\title{
Double-Peak Response in Orthodromic Sensory Nerve Conduction of the Median Nerve
}

\author{
Kyung Lim Joa, M.D. ${ }^{1,2}$, Chang-Hwan Kim, M.D. ${ }^{1}$ \\ Department of Physical \& Rehabilitation Medicine, 'School of Medicine, Inha University, Incheon 400-711, \\ ${ }^{2}$ Pusan National University School of Medicine, Yangsan 626-770, Korea
}

Objective To understand the neural generator of double-peak potentials and the change of latency and amplitude of double peaks with aging.

Method In 50 healthy subjects made up of groups of 10 people per decade from the age of 20 to 60 , orthodromic sensory nerve conduction studies were performed on the median nerves using submaximal stimulation. Various stimulus durations and interstimulation distances were used to obtain each double peak in the different age groups. The latency and amplitude of the second peak were measured. Statistical analyses included one-way ANOVA and correlation tests. $p$-values $<0.05$ were considered significant.

Results When the cathode moved in a proximal direction, the interpeak intervals increased. Second peak amplitudes decreased, and second peak latencies were delayed with aging $(\mathrm{p}<0.05)$. In some older people, second peaks were not obtained.

Conclusion Our experiments indicate that the double-peak response represented the two stimulation sites under the cathode and anode. The delayed latency and decreased amplitude of the second peak that occurs with aging represented peripheral nerve degeneration in aging, which starts at the distal nerve.

Key Words Nerve conduction study, Double peak response, Aging, Nerve degeneration

\section{INTRODUCTION}

During conventional orthodromic sensory nerve conduction studies (NCS) on the median nerve using surface electrodes, a sensory response characterized by two peaks is occasionally seen during incremental

Received May 17, 2010; Accepted March 14, 2011

Corresponding author: Chang-Hwan Kim

Department of Physical \& Rehabilitation Medicine, School of Medicine, Inha University, 7-206, Shinheung-dong 3ga, Joong-gu, Incheon 400-711, Korea

Tel: +82-32-890-2480, Fax: +82-32-890-2486, E-mail: jacob.kim@inha. ac.kr

(c) This is an open-access article distributed under the terms of the Creative Commons Attribution Non-Commercial License (http://creativecommons.org/ licenses/by-nc/3.0) which permits unrestricted noncommercial use, distribution, and reproduction in any medium, provided the original work is properly cited.

Copyright () 2011 by Korean Academy of Rehabilitation Medicine stimulation from minimal to slight strength. With stronger stimulation, the second peak disappeared, and a typical compound sensory action potential (CSAP) with a higher amplitude was recorded. ${ }^{1}$ According to Stalberg, this second peak originated from the skin receptors or intradermal nerve endings under the anode stimulation. ${ }^{1}$ A characteristic change in the aging peripheral nerve is that fiber-type grouping is more pronounced distally than proximally. ${ }^{2}$ The etiology of the fiber-type grouping was presumed secondary to degeneration and regeneration of the peripheral nervous system with secondary reorganization of the muscle fibers belonging to individual motor units. ${ }^{2}$ The density of large myelinated fibers in the distal portions of the sural nerve reaches a maximum fiber density of 6,480 fibers $/ \mathrm{mm}^{2}$ by the 
third decade of life. The large fiber density subsequently and progressively declines to $54 \%$ (3480 fibers $/ \mathrm{mm}^{2}$ ) from the second decade to 90 years of age. ${ }^{3}$ Another change in the peripheral nerve with the aging process is shortening of the internodal length, which can result from demyelination and remyelination. These changes are more pronounced distally in the peripheral nerve. ${ }^{4}$

With conventional NCS, far distal segments of sensory nerve fibers are not evaluated. The distal segments would reveal the nerve degeneration changes that occur with the aging process. In the present study, we decided to use the orthodromic double-peak response to detect changes in the far distal segments of peripheral nerves that occur with aging in normal subjects. The objective of this study was to understand the pathophysiologic changes that occur with aging and that progress from the far distal segment of the peripheral nerve, and to use the doublepeak response to detect peripheral neuropathy that starts at the distal nerve in a centripetal manner.

\section{MATERIALS AND METHODS}

\section{Subjects}

Subjects did not have diseases that may provoke peripheral neuropathy, such as diabetic neuropathy, alcoholism, stroke or spinal cord injury. We performed antidromic conduction studies of the median sensory nerve and excluded subjects with a latency of amplitude abnormality. A total of fifty subjects, with 10 people per decade from 20 to 60 (19 male, mean age: 22) were recruited. Approval for performing the NCS on healthy volunteers was granted by the institutional review board of Inha University Hospital. The subjects also provided

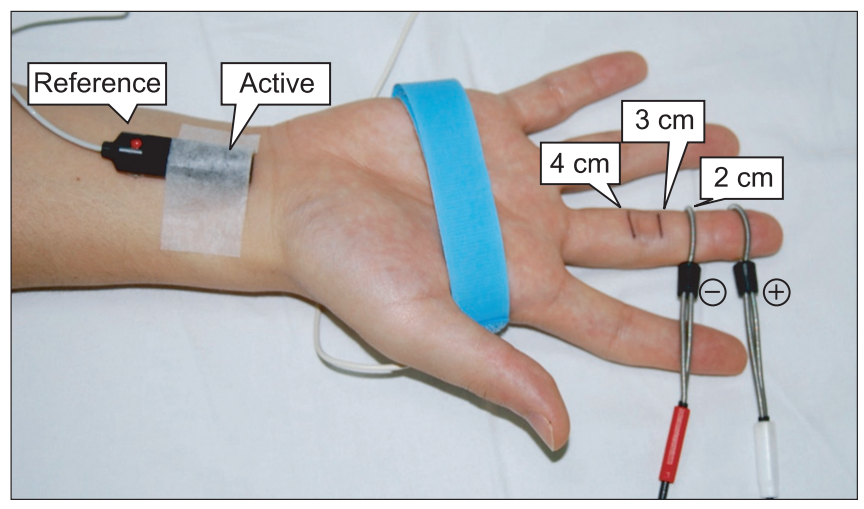

Fig. 1. Orthodromic stimulation of median sensory nerve on third finger tip. written informed consent.

\section{Methods}

Orthodromic NCS was performed with Medtronic Keypoint EMG equipment (Skovlunde, Denmark) in all subjects. The sweep speed was $1 \mathrm{~ms} /$ div, sensitivity was $10 \mu \mathrm{v} / \mathrm{div}$, and the filter setting was $20 \mathrm{~Hz}-2 \mathrm{kHz}$. Orthodromic stimulation was performed through a pair of ring electrodes that were 2,3 , or $4 \mathrm{~cm}$ apart, with the cathode proximally placed on the third digit for the median nerve. The bipolar recording of the sensory nerve action potential was performed with a surface bar electrode (Medtronic 9013L0221, 23 mm, Denmark, Medtronic) that was placed over the median nerve at the wrist (Fig. 1). A variety of stimulus durations were examined, including $0.1,0.2,0.3$, and $0.5 \mathrm{~ms}$, and at each stimulus duration, the stimulus intensity was progressively delivered through an increment of $0.2 \mathrm{~mA}$ until the double peak appeared. Intensity data of the initial appearance, the maximum, and the disappearance of the double-peak response were obtained. The latency and amplitude of the double peak was detected when the second peak amplitude was maximal, and the first and second double-peak latency and amplitude were obtained (Fig. 2). At each stimulus duration, the interstimulus distance was changed from 2,3 , and $4 \mathrm{~cm}$
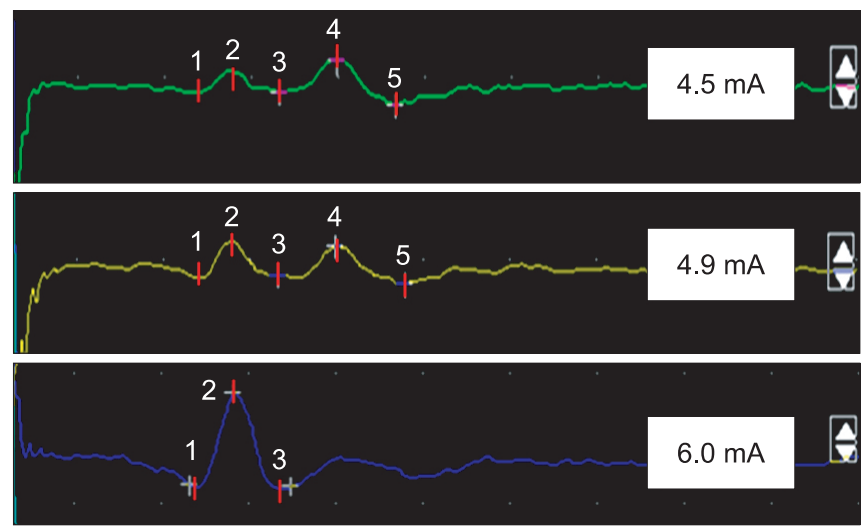

Fig. 2. Double-peak sensory nerve action potential at slightly increasing stimulation intensity at interstimuli interval of $4 \mathrm{~cm}$ and stimulation duration of $0.1 \mathrm{~ms}$. We measured the amplitude as the maximum peak-to-peak amplitude. For example, we detected the second peak amplitude of trace 2 (from 4 to 5 ). When supramaximal stimulation intensity reached the compound sensory action potential (CSAP) we obtained, we measured the amplitude of CSAP as maximum peak to-peak amplitude (from 2 to 3 ). 
apart to obtain the interpeak interval. Skin temperatures were constantly maintained at or above $31^{\circ} \mathrm{C}$ in the upper limbs.

\section{Statistical analysis}

Data was analyzed using SPSS (version 12.0; SPSS, Inc., Chicago, IL). The changes of the interpeak intervals with 2,3 , and $4 \mathrm{~cm}$ interstimulus distances were tested for statistical significance by one-way ANOVA tests. Pearson's correlation was used to examine the relationship between aging and other variables that consisted of the first and second peak latency and peak amplitude for both the $3 \mathrm{~cm}$ and $4 \mathrm{~cm}$ interstimulus distances and stimulus intensities. For all statistical analyses, $p$-value of less than 0.05 was considered statistically significant.

Table 1. Second Peak Latency with Interstimulus Distance $2 \mathrm{~cm}$ in Different Stimulation Duration at Each Age Group

\begin{tabular}{cccccc}
\hline & & \multicolumn{4}{c}{ Stimulation duration (ms) } \\
\cline { 2 - 6 } & & $\mathbf{0 . 1}$ & $\mathbf{0 . 2}$ & $\mathbf{0 . 3}$ & $\mathbf{0 . 5}$ \\
\hline Age (yrs) & $20-29$ & $3.98 \pm 0.15$ & $4.10 \pm 0.17$ & $4.21 \pm 0.19$ & $4.41 \pm 0.21$ \\
& $30-39$ & $4.10 \pm 0.41$ & $4.29 \pm 0.26$ & $4.40 \pm 0.31$ & $4.52 \pm 0.32$ \\
& $40-49$ & $4.51 \pm 0.38$ & $4.55 \pm 0.31$ & $4.69 \pm 0.32$ & $4.85 \pm 0.31$ \\
& $50-59$ & $4.50 \pm 0.31$ & $4.56 \pm 0.33$ & $4.74 \pm 0.29$ & $4.76 \pm 0.32$ \\
& $60-69$ & $4.73 \pm 0.27$ & $4.85 \pm 0.25$ & $4.91 \pm 0.26$ & $4.97 \pm 0.29$ \\
& & $\mathrm{r}=0.627$ & $\mathrm{r}=0.680$ & $\mathrm{r}=0.669$ & $\mathrm{r}=0.561$ \\
& $\mathrm{p}<0.01$ & $\mathrm{p}<0.01$ & $\mathrm{p}<0.01$ & $\mathrm{p}<0.01$ \\
\hline
\end{tabular}

Values are Mean \pm SD, Unit: $\mathrm{ms}$

Table 2. Second Peak Amplitude with Interstimulus Distance $2 \mathrm{~cm}$ in Different Stimulation Duration at Each Age Group

\begin{tabular}{cccccc}
\hline & & \multicolumn{4}{c}{ Stimulation duration (ms) } \\
\cline { 2 - 5 } & & $\mathbf{0 . 1}$ & $\mathbf{0 . 2}$ & $\mathbf{0 . 3}$ & $\mathbf{0 . 5}$ \\
\hline Age (yrs) & $20-29$ & $13.06 \pm 5.28$ & $13.03 \pm 4.65$ & $13.00 \pm 4.80$ & $12.68 \pm 4.74$ \\
& $30-39$ & $11.73 \pm 6.37$ & $11.99 \pm 5.89$ & $12.20 \pm 5.24$ & $11.69 \pm 5.01$ \\
& $40-49$ & $9.04 \pm 4.25$ & $8.73 \pm 4.20$ & $7.55 \pm 4.62$ & $8.38 \pm 4.4$ \\
& $50-59$ & $7.86 \pm 2.11$ & $7.61 \pm 2.17$ & $6.81 \pm 2.07$ & $7.66 \pm 2.76$ \\
& $60-69$ & $4.96 \pm 1.56$ & $6.06 \pm 1.33$ & $5.66 \pm 1.14$ & $4.74 \pm 1.13$ \\
& & $\mathrm{r}=-0.559$ & $\mathrm{r}=-0.545$ & $\mathrm{r}=-0.588$ & -0.602 \\
& $\mathrm{p}<0.01$ & $\mathrm{p}<0.01$ & $\mathrm{p}<0.01$ & $\mathrm{p}<0.01$ \\
\hline
\end{tabular}

Values are Mean \pm SD, Unit: $\mu \mathrm{V}$

Table 3. Second Peak Latency with Interstimulus Distance $3 \mathrm{~cm}$ in Different Stimulation Duration at Each Age Group

\begin{tabular}{cccccc}
\hline & & \multicolumn{4}{c}{ Stimulation duration (ms) } \\
\cline { 2 - 5 } & & $\mathbf{0 . 1}$ & $\mathbf{0 . 2}$ & $\mathbf{0 . 3}$ & $\mathbf{0 . 5}$ \\
\hline Age (yrs) & $20-29$ & $3.99 \pm 0.20$ & $4.11 \pm 0.19$ & $4.23 \pm 0.21$ & $4.39 \pm 0.20$ \\
& $30-39$ & $4.13 \pm 0.28$ & $4.23 \pm 0.28$ & $4.35 \pm 0.25$ & $4.51 \pm 0.30$ \\
& $40-49$ & $4.35 \pm 0.31$ & $4.20 \pm 0.92$ & $4.60 \pm 0.29$ & $4.73 \pm 0.35$ \\
& $50-59$ & $4.37 \pm 0.25$ & $4.54 \pm 0.30$ & $4.58 \pm 0.33$ & $4.83 \pm 0.37$ \\
& $60-69$ & $4.60 \pm 0.25$ & $4.74 \pm 0.20$ & $5.04 \pm 0.53$ & $5.00 \pm 0.22$ \\
& & $\mathrm{r}=0.640$ & $\mathrm{r}=0.642$ & $\mathrm{r}=0.631$ & $\mathrm{r}=0.585$ \\
& $\mathrm{p}<0.01$ & $\mathrm{p}<0.01$ & $\mathrm{p}<0.01$ & $\mathrm{p}<0.01$ \\
\hline
\end{tabular}

Values are Mean \pm SD, Unit: $\mathrm{ms}$ 


\section{RESULTS}

The relationship between aging and the double-peak response

The double-peak response was evoked in all subjects with an interstimulus interval of $4 \mathrm{~cm}$. However, in one 69-year-old subject, double-peak responses were not evoked with the $2 \mathrm{~cm}$ or $3 \mathrm{~cm}$ interstimulus intervals, and in one 54-year-old subject, it was not evoked with the $2 \mathrm{~cm}$ interstimulus interval. With increasing interstimulus intervals from $2 \mathrm{~cm}$ to $4 \mathrm{~cm}$, second peak latencies were delayed with increasing age except for the $0.5 \mathrm{~ms}$ stimulation duration (Table 1, 3, 5). Second peak amplitudes decreased with increasing age, regardless of the stimulation duration (Table 2, 4, 6). With the same interstimulus interval and stimulation duration, the stimulation intensity increased with aging to evoke maximal amplitude (Fig. 3; r=0.547, p<0.01).
The relationship between the anode and the cathode

With the $0.1 \mathrm{~ms}$ stimulation duration, the interpeak distance increased from $0.83,0.97$, and $1.15 \mathrm{~ms}$ with increasing interstimulus distances of 2,3 , and $4 \mathrm{~cm}$, respectively. With the $0.2,0.3$, and $0.5 \mathrm{~ms}$ interstimulus duration, the interpeak distance increased with interstimulus distance (Table 7).

\section{DISCUSSION}

During conventional orthodromic sensory NCS on the median nerve using surface electrodes, a sensory response characterized by two peaks was occasionally seen during the increase of stimulation from minimal to slight strength. With stronger stimulation, the second peak disappeared, and a typical sensory nerve action potential (SNAP) with a higher amplitude was recorded. ${ }^{1}$ Buchthal and Rosenfalck, ${ }^{5}$ using unipolar nerve recordings from the median nerve, obtained a response that consisted of two peaks. The authors believed that

Table 4. Second Peak Amplitude with Interstimulus Distance $3 \mathrm{~cm}$ in Different Stimulation Duration at Each Age Group

\begin{tabular}{cccccc}
\hline & & \multicolumn{4}{c}{ Stimulation duration $(\mathbf{m s})$} \\
\cline { 2 - 5 } & & $\mathbf{0 . 1}$ & $\mathbf{0 . 2}$ & $\mathbf{0 . 3}$ & $\mathbf{0 . 5}$ \\
\hline Age (yrs) & $20-29$ & $14.11 \pm 6.63$ & $14.35 \pm 8.20$ & $14.41 \pm 8.07$ & $13.72 \pm 7.42$ \\
& $30-39$ & $15.67 \pm 7.55$ & $14.61 \pm 7.34$ & $14.48 \pm 4.69$ & $13.35 \pm 4.21$ \\
& $40-49$ & $10.12 \pm 6.18$ & $10.06 \pm 6.23$ & $9.14 \pm 5.83$ & $9.01 \pm 5.45$ \\
& $50-59$ & $6.68 \pm 1.95$ & $8.39 \pm 3.88$ & $7.36 \pm 2.64$ & $8.02 \pm 3.24$ \\
& $60-69$ & $6.10 \pm 2.32$ & $5.98 \pm 2.85$ & $6.34 \pm 2.75$ & $5.48 \pm 1.64$ \\
& & $\mathrm{r}=-0.489$ & $\mathrm{r}=-0.546$ & $\mathrm{r}=-0.556$ & $\mathrm{r}=-0.563$ \\
& $\mathrm{p}<0.01$ & $\mathrm{p}<0.01$ & $\mathrm{p}<0.01$ & $\mathrm{p}<0.01$ \\
\hline
\end{tabular}

Values are Mean \pm SD, Unit: $\mu \mathrm{V}$

Table 5. Second Peak Latency with Interstimulus Distance $4 \mathrm{~cm}$ in Different Stimulation Duration at Each Age Group

\begin{tabular}{cccccc}
\hline & & \multicolumn{4}{c}{ Stimulation duration (ms) } \\
\cline { 2 - 5 } & & $\mathbf{0 . 1}$ & $\mathbf{0 . 2}$ & $\mathbf{0 . 3}$ & $\mathbf{0 . 5}$ \\
\hline Age (yrs) & $20-29$ & $3.93 \pm 0.20$ & $4.07 \pm 0.17$ & $4.18 \pm 0.20$ & $4.33 \pm 0.16$ \\
& $30-39$ & $4.05 \pm 0.28$ & $4.16 \pm 0.23$ & $4.27 \pm 0.28$ & $4.43 \pm 0.31$ \\
& $40-49$ & $4.37 \pm 0.36$ & $4.50 \pm 0.30$ & $4.59 \pm 0.31$ & $4.37 \pm 1.47$ \\
& $50-59$ & $4.27 \pm 0.29$ & $4.34 \pm 0.28$ & $4.43 \pm 0.33$ & $4.54 \pm 0.35$ \\
& $60-69$ & $4.43 \pm 0.44$ & $4.65 \pm 0.24$ & $4.74 \pm 0.24$ & $4.87 \pm 0.31$ \\
& & 0.491 & 0.594 & 0.537 & 0.270 \\
& $\mathrm{p}<0.01$ & $\mathrm{p}<0.01$ & $\mathrm{p}<0.01$ & $\mathrm{p}=0.06$ \\
\hline
\end{tabular}

Values are Mean \pm SD, Unit: $\mathrm{ms}$ 
Table 6. Second Peak Amplitude with Interstimulus Distance $4 \mathrm{~cm}$ in Different Stimulation Duration at Each Age Group

\begin{tabular}{cccccc}
\hline & & \multicolumn{4}{c}{ Stimulation duration (ms) } \\
\cline { 2 - 5 } & & $\mathbf{0 . 1}$ & $\mathbf{0 . 2}$ & $\mathbf{0 . 3}$ & $\mathbf{0 . 5}$ \\
\hline Age (yrs) & $20-29$ & $17.99 \pm 7.32$ & $20.10 \pm 6.94$ & $19.37 \pm 7.29$ & $15.61 \pm 6.28$ \\
& $30-39$ & $16.62 \pm 8.50$ & $17.91 \pm 9.43$ & $16.33 \pm 3.12$ & $15.79 \pm 8.34$ \\
& $40-49$ & $10.99 \pm 6.72$ & $12.29 \pm 6.22$ & $10.84 \pm 6.19$ & $10.62 \pm 6.72$ \\
& $50-59$ & $9.36 \pm 3.65$ & $9.64 \pm 4.15$ & $9.23 \pm 4.20$ & $11.36 \pm 4.58$ \\
& $60-69$ & $6.05 \pm 1.51$ & $7.46 \pm 2.10$ & $6.10 \pm 1.86$ & $9.74 \pm 2.64$ \\
& & -0.627 & -0.630 & -0.713 & -0.425 \\
& & $\mathrm{p}<0.01$ & $\mathrm{p}<0.01$ & $\mathrm{p}<0.01$ & $\mathrm{p}<0.01$ \\
\hline
\end{tabular}

Values are Mean \pm SD, Unit: $\mu \mathrm{V}$

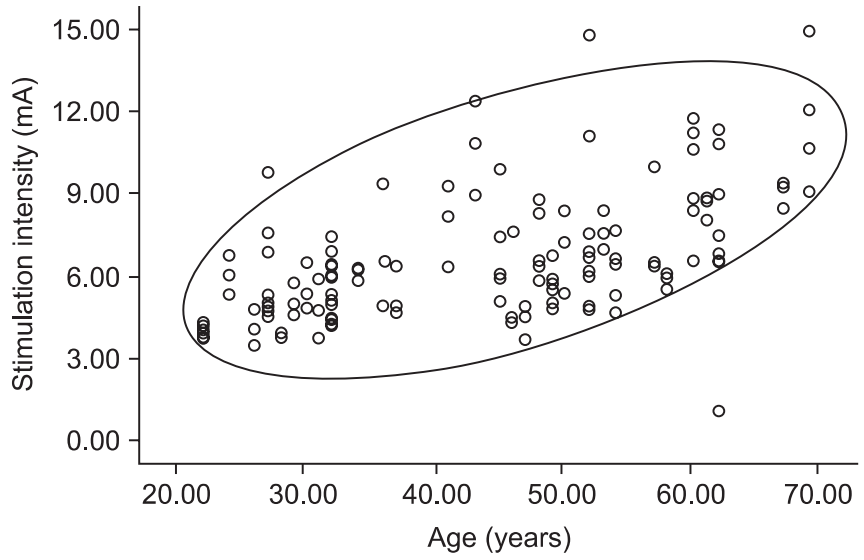

Fig. 3. There was a significant positive relationship between age (year) and stimulation intensity $(\mathrm{mA})(\mathrm{r}=0.547, \mathrm{p}<0.01)$ by Pearson's correlation test.

the particular sensory response that was evoked with submaximal stimulus was the expression of the fast and slow fibers. ${ }^{5}$ However, Stalberg ${ }^{1}$ measured a double-peak response at the digit-wrist and wrist-elbow segments. The sensory nerve conduction velocity (SNCV) was measured for the two peaks separately, and the SNCV of the first and second peaks did not differ. He concluded that the first and second peak originated from the same nerve. In his study, orthodromic stimulation was performed through a pair of ring electrodes with the cathode placed proximally on the third digit for the median nerve. With an increasing interstimulus distance, the increment of the interpeak interval of the double-peak response was found, and he concluded that the double peak originated from the two stimuli, or the anode and the cathode. ${ }^{1}$ He also found a delay in the first peak latency when the double-peak response was obtained with the anode
Table 7. Inter-peak Intervals Change with Interstimulus Distance Among Different Duration of Stimulation

\begin{tabular}{cccc}
\hline \multirow{2}{*}{$\begin{array}{c}\text { Duration of } \\
\text { stimulation }\end{array}$} & \multicolumn{3}{c}{ Interstimulus distance } \\
\cline { 2 - 4 } & $\mathbf{2} \mathbf{~ c m}$ & $\mathbf{3 ~ c m}$ & $\mathbf{4} \mathbf{~ c m}$ \\
\hline $0.1 \mathrm{~ms}$ & $0.83 \pm 0.17$ & $0.97 \pm 0.17$ & $1.15 \pm 0.21$ \\
$0.2 \mathrm{~ms}$ & $0.85 \pm 0.15$ & $0.98 \pm 0.40$ & $1.22 \pm 0.16$ \\
$0.3 \mathrm{~ms}$ & $0.90 \pm 0.15$ & $1.09 \pm 0.26$ & $1.26 \pm 0.23$ \\
$0.5 \mathrm{~ms}$ & $0.91 \pm 0.21$ & $1.08 \pm 0.18$ & $1.17 \pm 0.60$ \\
\hline
\end{tabular}

Values $=$ Mean \pm SD, Unit: $\mathrm{ms}$

$\mathrm{p}<0.05$, by ANOVA

proximal and the cathode in the distal position, and he explained the cause as an anodal delay. This anodal delay may be evoked by anodal stimulation of the skin sensory receptor or intradermal nerve endings, which are slowed by receptorial delay. ${ }^{1}$

Capsaicin can induce chemical denervation of epidermal nerve fibers. ${ }^{6,7}$ Simone and co-workers showed that an intradermal injection of capsaicin produced a decreased sensitivity to pain from mechanical stimuli and nearly eliminated heat evoked pain. These symptoms resulted from rapid degeneration of intracutaneous nerve fibers. ${ }^{8}$ After capsaicin application to the distal middle finger, there was a reduction in the second peak amplitude of the double-peak response, which suggested a reduction in the functions of skin receptors and intradermal nerve endings. In addition, this finding also showed that the second peak originated from the distal anodal stimulation in orthodromic sensory stimulation. ${ }^{9}$ This second peak amplitude change is compatible with our data showing second peak amplitude decrement with aging. In addition, we can suspect that the subtle 
degeneration change that occurs with aging starts from the far distal endings of the peripheral nerve, and this can be diagnosed with the orthodromic double-peak response.

Recent studies on the morphology of the distal peripheral nervous system showed that cutaneous myelinated nerve fibers lose their myelination before their entrance into the dermis and connection to Meissner's corpuscle. The classic criteria, which is based on fiber size (small and large), used to distinguish myelinated fiber populations in sensory nerves no longer seems suitable for identifying myelinated terminations in the skin. ${ }^{10}$ In fact, instead of a significant variation of fiber diameter along nerves between proximal and distal tapering of nerve fibers, a significant proximal-distal reduction of the internodal length along the nerve trunk was observed. These more recent acquisitions provide evidence that the second peak, which is a result of anodal stimulation, has an initial delay that could be due to a progressive shortening and reduced intermodal length of cutaneous myelinated fibers. ${ }^{11}$

Histochemical studies of limb muscles from elderly individuals (65 years or older) without evidence of neuromuscular disease revealed fiber size variation, hyaline or granular degeneration, loss of striations, clumps of pyknotic nuclei, increased fat and connective tissue, and most significantly, neurogenic fiber-type grouping. ${ }^{2,12}$ In addition, there were decreased internodal distances and loss of myelin from sensory myelinated fibers innervating the distal portions of the peripheral nerve. A lessening of the internodal length can result from demyelination and remyelination. ${ }^{2-4}$ Sensory nerve conduction velocities demonstrate a consistent decline that of nearly 1-2 meters/second per decade. ${ }^{13,14}$ Compared to the 18-25-year-old group, the SNAP's amplitude is one-half and one-third, respectively, for the 40-60- and 70-88-year-old group. ${ }^{5}$ The NCS findings may be explained to some degree by considering the histologic changes associated with the aging of the peripheral nervous system. The decrement of amplitude of sensory NCS was demonstrated not only with a cross-sectional study, but also with a prospective study of orthodromic stimulation of the median nerve. In this study, an increment of latency and decrement of conduction velocity was also noted. ${ }^{15,16}$

These changes of the peripheral nervous system with aging are prominent at far distal areas. However, with conventional NCS, these subtle changes cannot be found. With the orthodromic double-peak response, we can evaluate the distal peripheral nerve. In our study, with the same inter-stimulus distance and duration, there was a decrement of amplitude and an increment of latency of the second peak response. In addition, we found a relationship between stimulus intensity and aging. In some old people, we could not obtain the double-peak response. These changes of the doublepeak response represent degeneration and loss of nerve ending receptors in the distal peripheral nerve. In some older age groups, we could not induce the double-peak response with inter-stimulus distances of $2 \mathrm{~cm}$ or $3 \mathrm{~cm}$, but with the $4 \mathrm{~cm}$ interstimulus distance, we could obtain the double-peak response in all subjects. This finding supports the importance of interstimulus distance. With shorter inter-stimulus distances, there might be an anodal block or refractory period of anodal peak.

One limitation of our study was the small number of subjects in each age group. Especially in the older age groups, there were non-responders. Like the H reflex, which is equivalent to ankle jerk, it is accepted as normal when this cannot be elicited in subjects older than 65 years. Therefore, we did not know the exact meaning of no response in the very old age groups. It is possible that these non-responders represent early length-dependent neuropathies. Secondly, double-peak responses were obtained with upper extremity sensory stimulations only, and so the effects of aging on the motor nerve and lower extremity nerves were not investigated in our experiment. Further study is needed on the double-peak responses in age groups over 70 to understand the differences between aging and disease processes in the very old age group.

\section{CONCLUSION}

There was an increment of amplitude, latency, and stimulus intensity and a decrement of conduction velocity of the double peak-response with orthodromic median NCS in normal healthy subjects with aging. In some older aged people, double-peak responses were not obtained.

With the assumption that far distal nerve ending degeneration can be explained with the second peak of the double-peak response, we can use the double-peak 
response as a screening tool for degenerative peripheral neuropathy with aging, and even for metabolic or toxic peripheral neuropathy with reduced time and cost.

\section{ACKNOWLEDGEMENTS}

This work was supported by Inha University research grant.

\section{REFERENCES}

1. Aprile I, Stalberg E, Tonali P, Padua L. Double peak sensory responses at submaximal stimulation. Clin Neurophysiol 2003; 114: 256-262

2. Jennekens FG, Tomlinson BE, Walton JN. Histochemical aspects of five limb muscles in old age. An autopsy study. J Neurol Sci 1971; 14: 259-276

3. Tohgi H, Tsukagoshi H, Toyokura Y. Quantitative changes with age in normal sural nerves. Acta Neuropath 1977; 38: 213-220

4. Lascelles RG, Thomas PK. Changes due to age in intermodal length in the sural nerve in man. J Neurol Neurosurg Psychiatry 1966; 29: 40-44

5. Buchthal F, Rosenfalck A. Evoked action potentials and conduction velocity in human sensory nerves. Brain Res 1966; 3: 74-78

6. Nolano M, Simone DA, Wendelschafer-Crabb G, Johnson T, Hazen E, Kennedy WR. Topical capsaicin in humans: parallel loss of epidermal nerve fibers and pain sensation. Pain 1999; 81: 135-145

7. Lynn B. Capsaicin: actions on nociceptive C-fibers and therapeutic potential. Pain 1990; 41: 61-69

8. Simone DA, Nolano M, Johnson T, Simone Da, Nolano M, Johnson T, Wendelschafer-Crabb G, Kennedy
WR. Intradermal injection of capsaicin in humans produces degeneration and subsequent reinnervation of epidermal nerve fibers: correlation with sensory function. J Neurosci 1998; 18: 8947-8959

9. Aprile I, Tonali P, Stalberg E, Di Stasio E, Caliandro P, Foschini M, Vergili G, Padua L. Double peak sensory responses: effects of capsaicin. Neurol Sci 2007; 28: 264-269

10. Provitera V, Nolano M, Pagano A, Caporaso G, Stancanelli A, Santoro L. Myelinated nerve endings in human skin. Muscle Nerve 2007; 35: 767-775

11. Nolano M, Provitera V, Crisci C, Stancanelli A, Wendelschafer-Crabb G, Kennedy WR, Santoro L. Quantification of myelinated endings and mechanoreceptors in human digital skin. Ann Neurol 2003; 54: 197-205

12. Dumitru D, Amato AA, Zwarts M. Nerve conduction studies. In: Dumitru D, Editor. Electrodiagnostic medicine, 2nd ed, Philadelphia, Hanley \& Belfus Inc, 2002, 187

13. Dumitru D, Amato AA, Zwarts M. Nerve conduction studies. In: Dumitru D, Editor. Electrodiagnostic medicine, 2nd ed, Philadelphia, Hanley \& Belfus Inc, 2002, 188

14. Bolton CF, Carter KM. Human sensory nerve compound action potential amplitude: variation with sex and finger circumference. J Neurol Neurosurg Psychiatry 1980; 43: 925-928

15. Tong HC, Werner RA, Franzblau A. Effect of aging on sensory nerve conduction study parameters. Muscle Nerve 2004; 29: 716-720

16. Rivner MH, Swift TR, Malik K. Influence of age and height on nerve conduction. Muscle Nerve 2001; 24: 1134-1141 